\title{
Comparison study of intensity modulated arc therapy using single or multiple arcs to intensity modulated radiation therapy for high-risk prostate cancer
}

\author{
Hani Ashamalla, MD ${ }^{1,2}$, Ajay Tejwani, MD, MPH ${ }^{1,2}$, Ioannis Parameritis, MSc ${ }^{2}$ Uma Swamy, MD', \\ Pei Ching Luo, MSc ${ }^{2}$, Adel Guirguis, MD1, Amir Lavaf, MD ${ }^{1,2}$ \\ 'New York Methodist Hospital, Brooklyn, NY; ${ }^{2}$ Leading Edge Radiation Oncology (LEROS), \\ Weill Medical College of Cornell University, Brooklyn, NY, USA
}

Purpose: Intensity modulated arc therapy (IMAT) is a form of intensity modulated radiation therapy (IMRT) that delivers dose in single or multiple arcs. We compared IMRT plans versus single-arc field (1ARC) and multi-arc fields (3ARC) IMAT plans in high-risk prostate cancer.

Materials and Methods: Sixteen patients were studied. Prostate (PTV $)$, right pelvic $\left(\mathrm{PTV}_{\text {RtIN }}\right)$ and left pelvic lymph nodes $\left(\mathrm{PTV}_{\text {lttN)}}\right)$ and organs at risk were contoured. PTV $\mathrm{P}_{1}$ PTV $V_{\text {RtIN }}$ and $\mathrm{PTV}_{\text {LtIN }}$ received $50.40 \mathrm{~Gy}$ followed by a boost to PTV $\mathrm{PF}_{B} 28.80 \mathrm{~Gy}$. Three plans were per patient generated: IMRT, $1 A R C$, and $3 A R C$. We recorded the dose to the PTV, the mean dose ( $\left.D_{\text {MEAN }}\right)$ to the organs at risk, and volume covered by the $50 \%$ isodose. Efficiency was evaluated by monitor units (MU) and beam on time (BOT). Conformity index (CI), Paddick gradient index, and homogeneity index (HI) were also calculated.

Results: Average Radiation Therapy Oncology Group Cl was 1.17, 1.20, and 1.15 for IMRT, 1ARC, and 3ARC, respectively. The plans' $\mathrm{HI}$ were within $1 \%$ of each other. The $\mathrm{D}_{\text {MEAN }}$ of bladder was within $2 \%$ of each other. The rectum $\mathrm{D}_{\text {MEAN }}$ in IMRT plans was $10 \%$ lower dose than the arc plans $(p<0.0001)$. The $\mathrm{GI}$ of the 3 ARC was superior to IMRT by $27.4 \%(p=0.006)$. The average MU was highest in the IMRT plans (1686) versus IARC (575) versus 3ARC (1079). The average BOT was 6 minutes for IMRT compared to 1.3 and 2.9 for IARC and 3ARC IMAT $(p<0.05)$.

Conclusion: For high-risk prostate cancer, IMAT may offer a favorable dose gradient profile, conformity, MU and BOT compared to IMRT.

Keywords: High risk prostate cancer, Intensity modulated arc therapy, Intensity modulated radiation therapy, RapidArc

\section{Introduction}

Prostate cancer is a site in which highly conformal therapies have gained widespread use secondary to the organs at risk in the area and the benefit seen with dose escalation in meta-analysis [1]. In many large centers, intensity modulated radiation therapy (IMRT)-based plans have become standard for patients with prostate cancer. Volumetric modulated arc therapy (VMAT) is a specific form of IMRT, which has recently gained popularity. This technique delivers radiation dose

Received 23 April 2013, Revised 6 June 2013, Accepted 10 June 2013.

Correspondence: Ajay Tejwani, MD, MPH, New York Methodist Hospital, 506 Sixth Street, Brooklyn, NY 11215, USA. Tel: +1 718-780-3677, Fax: +1-718-780-3688, E-mail: ajaytejwani@gmail.com

Presented at the American Society of Therapeutic Radiation Oncology (ASTRO) meeting 2010, San Diego, CA, S362.

(c) This is an Open Access article distributed under the terms of the Creative Commons Attribution Non-Commercial License (http://creativecommons.org/ licenses/by-nc/3.0/) which permits unrestricted non-commercial use, distribution, and reproduction in any medium, provided the original work is properly cited.

www.e-roj.org 
with up to 360-degree gantry rotations while the multileaf collimators (MLCs) transition at various angles. The radiation can be delivered at a constant dose rate (cdr-VMAT) or a variable dose rate (vdr-VMAT). Arc therapy can be delivered as multiple or single arcs. Single arc-modulated radiation therapy (AMRT) delivers dose in a single gantry rotation and multiarc intensity-modulated arc therapy (IMAT) uses multiple overlapping arcs. The advantage of IMAT is its highly conformal dose distribution [2], whereas AMRT is more efficient, delivering the same dose in a shorter period of time [3].

In this study we compare IMRT, to $1 A R C$, and 3ARC IMAT (RapidArc; Varian Medical Systems, Palo Alto, CA, USA) to address these issues. Specifically, we studied sixteen patients with high-risk prostate cancer as defined by the National Comprehensive Cancer Network (NCCN). Our goal is to quantify the target volume adequacy of coverage and efficiency as well as dose to organs at risk (OARs). Additionally, we compare results from low dose volume of radiation for each modality.

\section{Materials and Methods}

Sixteen patients with high-risk prostate cancer (prostatespecific antigen $[P S A]>20$ and/or Gleason score $\geq 8$ ) were included in this study. The study was approved by our Institutional Review Board. Computed tomography (CT) scan simulation was acquired for all patients and the images were imported to the Eclipse treatment planning station (ver. 8.6, Varian Medical Systems). The radiation oncologist contoured the prostate and seminal vesicles $\left(\mathrm{CTV}_{P}\right)$ as well as the pelvic lymph nodes based on CT images. The corresponding PTVS were created by adding a $1-\mathrm{cm}$ margin around the pelvic lymph nodes in all directions, and $0.7 \mathrm{~cm}$ around the $\mathrm{CTV}_{p}$ in all directions except the posterior margin where a $0.5 \mathrm{~cm}$ margin was used. $A \mathrm{PTV}_{B}$ was also created by expanding the $\mathrm{CTV}_{P}$ by $0.6 \mathrm{~cm}$ in all directions except the posterior margin where a 0.5 $\mathrm{cm}$ margin was used.

Rectum, bladder, small bowel, and acetabulum were contoured as the OARs. The rectum was contoured starting at the level of ischial tuberosities to the rectosigmoid junction. The urinary bladder was contoured based on the CT simulation images. Similarly, the small bowel loops were contoured based on the CT images to $3 \mathrm{~cm}$ superior to the top slice of the PTV . Total planned dose was $79.20 \mathrm{~Gy} / 1.8 \mathrm{~Gy} /$ fraction. The PTV RtLN $_{1}$ PTV ${ }_{L t L N \text {, }}$ and initial PTV received $50.4 \mathrm{~Gy} / 1.8 \mathrm{~Gy} /$ fraction followed by a 1 ARC boost of 28.80 Gy to PTV $V_{B}$. This study reports only on the initial dose of 50.40 Gy to the first 3
Table 1. Comparison between the plans, based on the p-value score

\begin{tabular}{lccc}
\hline & $\begin{array}{c}\text { IMRT vs. } \\
\text { 1ARC }\end{array}$ & $\begin{array}{c}\text { IMRT vs. } \\
\text { 3ARC }\end{array}$ & $\begin{array}{c}\text { 1ARC vs. } \\
\text { 3ARC }\end{array}$ \\
\hline "Beam ON" time & $0.0001(-)$ & $0.0010(-)$ & $0.0001(+)$ \\
Monitor unit & $0.0001(-)$ & $0.0001(-)$ & $0.0001(+)$ \\
V $_{50}$ & $0.0010(-)$ & $0.0001(-)$ & $0.0074(-)$ \\
D $_{\text {MEAN }}$ bladder & $1.0000(C)$ & $1.0000(C)$ & $1.0000(C)$ \\
D $_{\text {MEAN }}$ rectum & $0.0010(+)$ & $0.0001(+)$ & $0.6072(\mathrm{C})$ \\
Homogeneity index & $0.3018(\mathrm{C})$ & $0.3018(\mathrm{C})$ & $0.6072(\mathrm{C})$ \\
Conformity index & $0.0352(-)$ & $0.6072(\mathrm{C})$ & $0.3018(\mathrm{C})$ \\
Gradient index & $0.0001(-)$ & $0.0010(-)$ & $0.0010(-)$ \\
\hline
\end{tabular}

The sign next to the number indicates that for the specific parameter, the first plan is to the second: (+), better; (-), worse; or (C), comparable.

IMRT, intensity modulated radiation therapy; $V_{50}$, intergral dose; $D_{\text {MEAN }}$ mean dose.

PTVs because of the inclusion of the lymph nodes in the target volume and the theoretical benefit of the arc-modulated therapy in this setting.

Data were collected and summarized. Two-sided sign tests have been conducted to compare between the three plans, two at a time (Table 1), p-value of $<0.05$ is considered significant. STATA statistical software package ver. 12.0 (STATA Corp., College Station, TX, USA) has been used for the analysis.

\section{Treatment planning}

All treatments were planned using the Eclipse treatment planning system, and delivered by the iX linear accelerator (Varian Medical Systems). Three plans were generated per patient, as illustrated in (Fig. 1).

1) An IMRT plan with one isocenter and 7 static fields. The fields were evenly distributed every $51.5^{\circ}$, starting from the anterior field. To minimize the dose to the rectum, the lower two posterior oblique fields were designed to spare the organ by partially covering the target, and they had a fixed size during the optimization. The MLC motion was optimized using the sliding window technique, resulting in a slightly higher number of monitor units (MUs) and a significantly lower beam on time (BOT). The dose rate was equal to $300 \mathrm{MU} / \mathrm{min}$.

2) A one field ARC plan, with the beam performing a full $360^{\circ}$ rotation around the single isocenter. The gantry rotated from $179.9^{\circ}$ to $180.1^{\circ}$. The dose rate was variable with a maximum value of $600 \mathrm{MU} / \mathrm{min}$ (averaging around $300 \mathrm{MU} / \mathrm{min}$ ).

3) A three coplanar fields ARC plan. Each field had a separate isocenter, each one located at the center of the corresponding 

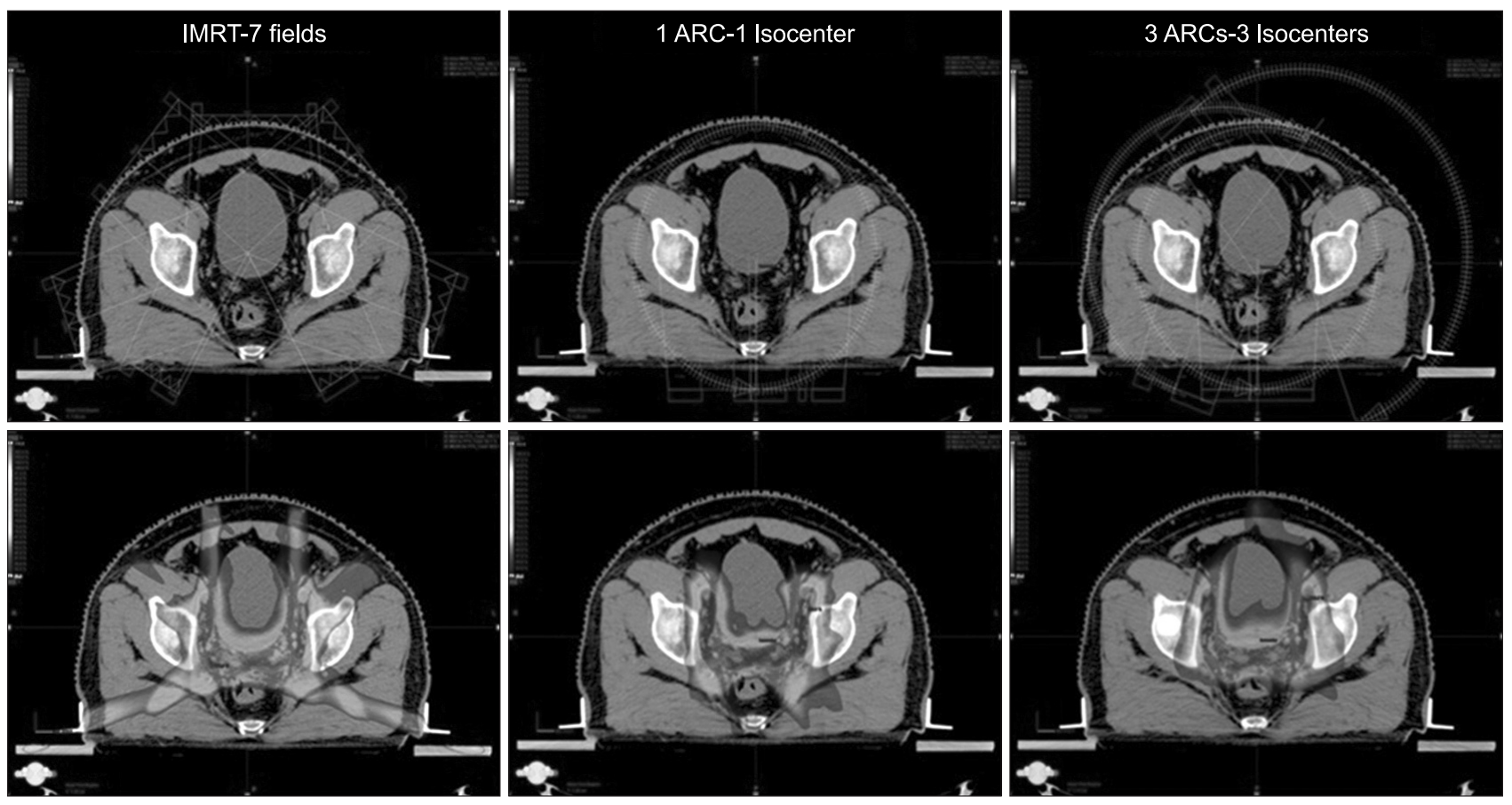

Fig. 1. Field arrangement and dose distribution for $V_{50} \leq$ dose $\leq V_{\operatorname{Max}}$.

PTV. The gantry performed a full $360^{\circ}$ rotation around PTV $_{P_{1}}$

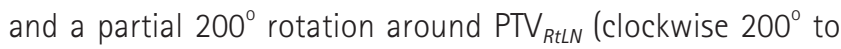
$40^{\circ}$ ) and $\mathrm{PTV}_{\text {LtLN }}$ (counter clockwise $40^{\circ}$ to $200^{\circ}$ ). The dose rate was variable with a maximum value of $600 \mathrm{MU} / \mathrm{min}$ (averaging around $300 \mathrm{MU} / \mathrm{min}$ ).

All plans were optimized using a standard planning constraint set based on the Quantitative Analyses of Normal Tissue Effects in the Clinic (QUANTEC) [4] and Radiation Therapy Oncology Group (RTOG) protocols [5], dose objectives and priorities. All three plans maintained the maximum dose $D_{\text {MAX }}$ $\leq 110 \%$, and were normalized to ensure 95\% coverage for the total PTV $_{T}\left(\right.$ PTV $_{P}+$ PTV $V_{\text {RtLN }}+$ PTV $\left._{L t L N}\right)$.

\section{Plan evaluation}

Plans were evaluated in terms of quality and efficiency. For this purpose, we recorded the monitor units, beam on time, the mean dose $\left(D_{\text {MEAN }}\right)$ to the OARs, and the volume covered by the $50 \%$ isodose line $\left(V_{50}\right)$.

Treatment efficiency was evaluated based on the comparison of the:

- MU defined as the average number of monitor units to required deliver the prescribed dose.

- BOT defined as the time the treatment planning system (Eclipse, Varian Medical Systems) predicts the beam will be "on" to deliver the prescribed monitor units. Does not account for time required to reach each gantry position; as in the case of IMRT.

Treatment quality included the comparison of the mean dose $\left(D_{\text {MEAN }}\right)$ to the OAR such as the bladder and the rectum, the volume covered by the $50 \%$ isodose line $\left(V_{50}\right)$, and the following indexes:

- Conformity index $(\mathrm{CI})$ calculated according to the RTOG index score definition: $\mathrm{Cl}=\mathrm{PV}_{95 \%} / \mathrm{PTV}$, where $\mathrm{PV}_{95 \%}$ is the volume that received the effective prescribed dose $(95 \%$ in this study), and PTV is the planned treatment volume.

- Paddick gradient index (GI), defined as $\mathrm{Cl}=\mathrm{PV}_{50 \%} / \mathrm{PV}$, where $\mathrm{PV}_{50 \%}$ is the volume that received $50 \%$ of the effective prescribed dose, and PV the prescribed dose $\left(\mathrm{PV}_{95 \%}\right.$ in the study).

- Homogeneity index (HI) calculated as $\mathrm{HI}=\mathrm{I}_{\text {MAX }} / \mathrm{RI}$, where $\mathrm{I}_{\text {MAX }}$ is the maximum isodose in the target and $\mathrm{Rl}$ is the reference isodose (100\% in this study).

For each one of the above parameters, the average value and the standard deviation (SD) were calculated. Plans were reviewed for dose distribution, OAR sparing, and integral dose to surrounding normal tissue. All of them satisfied the planning objectives, and considered acceptable for treatment. 
A

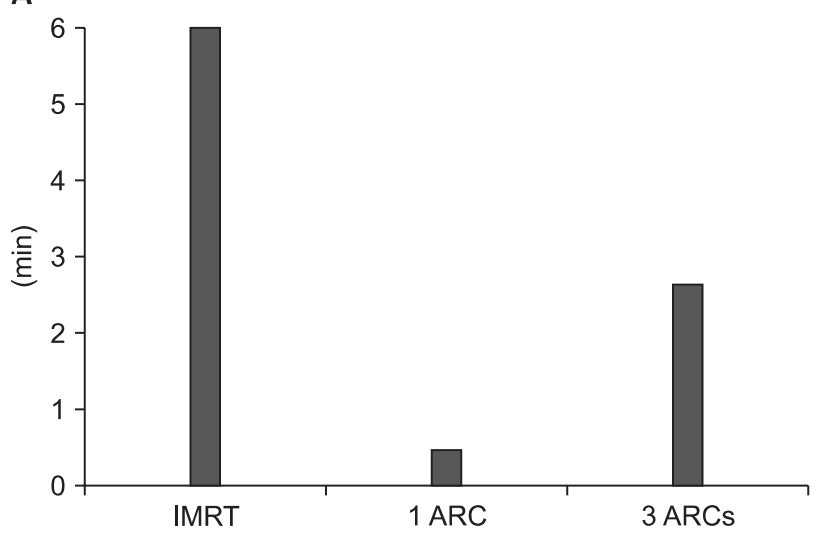

B

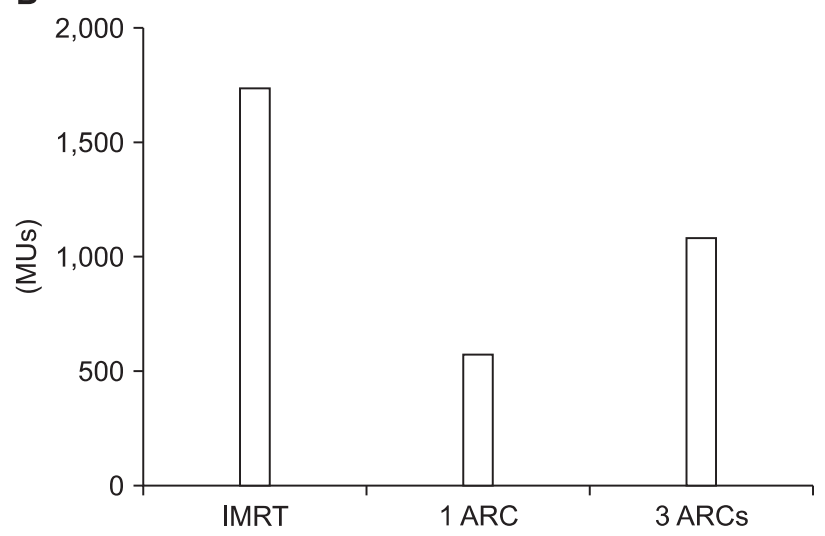

Fig. 2. (A) Average "Beam On" time in minutes and (B) average number of monitor units (MU). IMRT, intensity modulated radiation therapy.

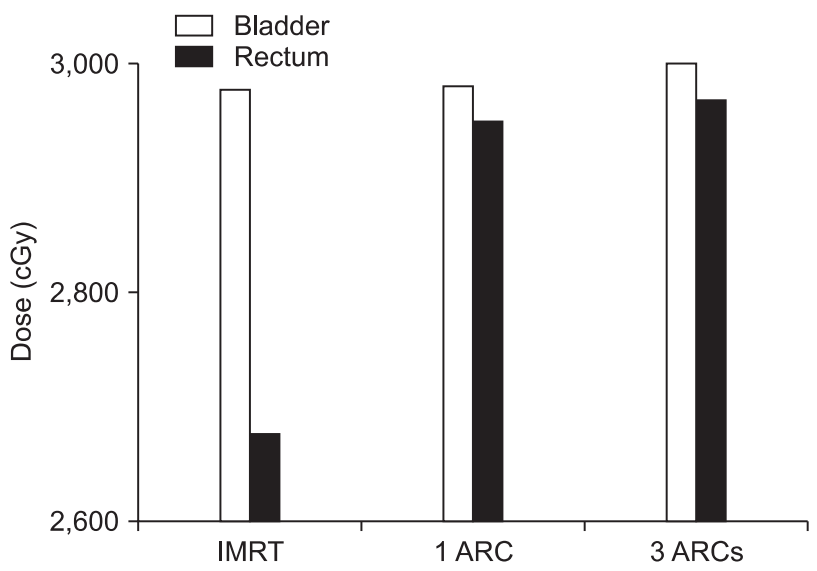

Fig. 3. Average $D_{\text {MEAN }}$ of rectum and bladder. IMRT, intensity modulated radiation therapy.

\section{Results}

\section{Plan efficiency}

BOT: The BOT decreased with the IMAT plans compared to IMRT. The shortest time was achieved with 1ARC. The average BOT for IMRT, 1ARC and 3ARC plans were 6.0, 1.4, and 3.2 minutes, respectively (Fig. 2A). The range (and SD) was 4.757.55 (0.75), 1.25-1.86 (0.28), and 2.63-3.59 (1.03), respectively.

MU: The MU decreased significantly with the IMAT plans compared to IMRT; lowest number was achieved with 1ARC. The average MU was 1,739, 576, and 1,086 for IMRT, 1ARC, and $3 A R C$ plans, respectively (Fig. 2B). The range (SD) was 9562,260 (264), 481-648 (51), and 644-1,345 (166), respectively.

\section{Plan quality}

Mean dose $\left(D_{\text {MEAN }}\right)$ for OAR: Rectal $D_{\text {MEAN }}$ was lower with IMRT

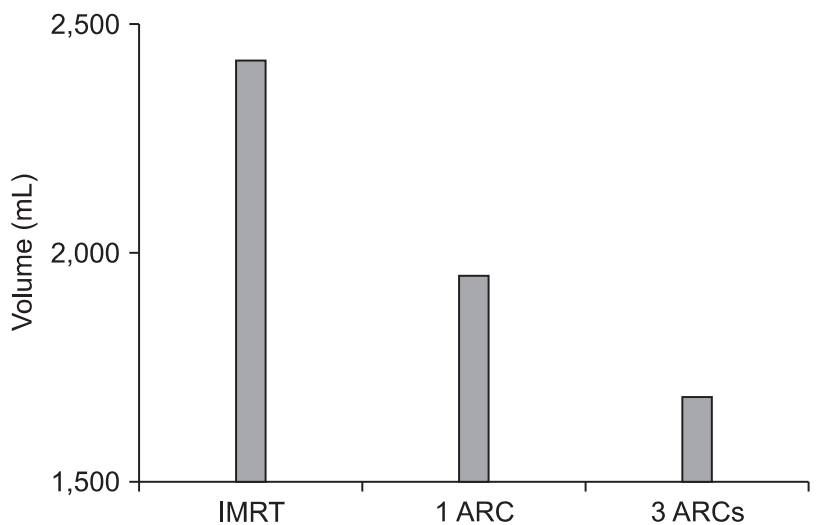

Fig. 4. Average integral dose $\left(V_{50}\right)$. IMRT, intensity modulated radiation therapy.

in comparison to IMAT plans and was comparable between IARC and 3ARCs. There was no significant difference between the three plans as far as the $D_{\text {MEAN }}$ of bladder (Fig. 3).

Integral dose $\left(V_{50}\right)$ : The volume covered by the $50 \%$ isodose line was significantly higher with the IMRT plan in comparison to the IMAT plans. The average $\mathrm{V}_{50}$ (in $\mathrm{mL}$ ) among the three plans was 2,423, 1,952, and 1,685 for IMRT, 1ARC, and 3ARC, respectively (Fig. 4).

$\mathrm{Cl}$ : The $\mathrm{Cl}$ was comparable among the plans. The average $\mathrm{Cl}$ among the three plans was 1.16, 1.19, and 1.15, respectively (Fig. 5). The 1ARC plan was significantly better than IMRT ( $p=$ 0.0352), none of the other plans were significant (NS).

GI: The average GI was 7.54, 5.93, and 5.24 for IMRT, 1ARC, and 3ARC plans, respectively (Fig. 6). There improvement was significant when using IMAT compared to IMRT. It was also decreased significantly when $3 A R C$ was compared to $1 A R C$.

$\mathrm{HI}$ : There was no significant difference amongst the three 


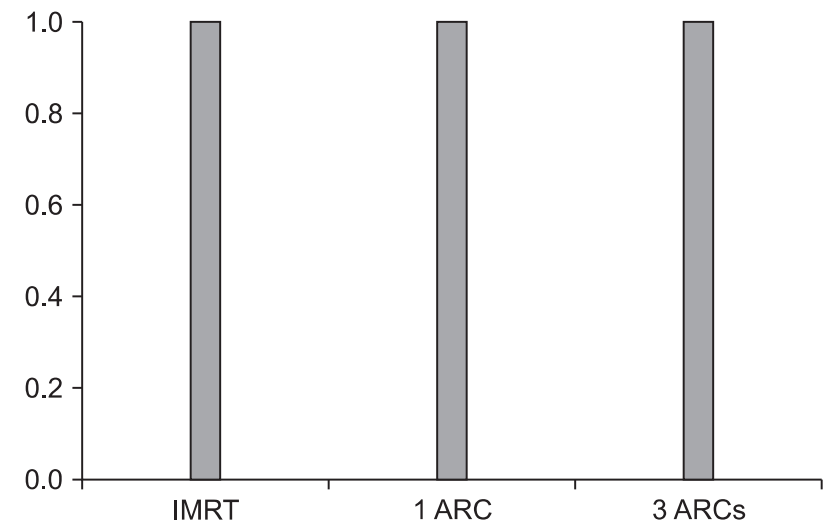

Fig. 5. Average conformity index. IMRT, intensity modulated radiation therapy.

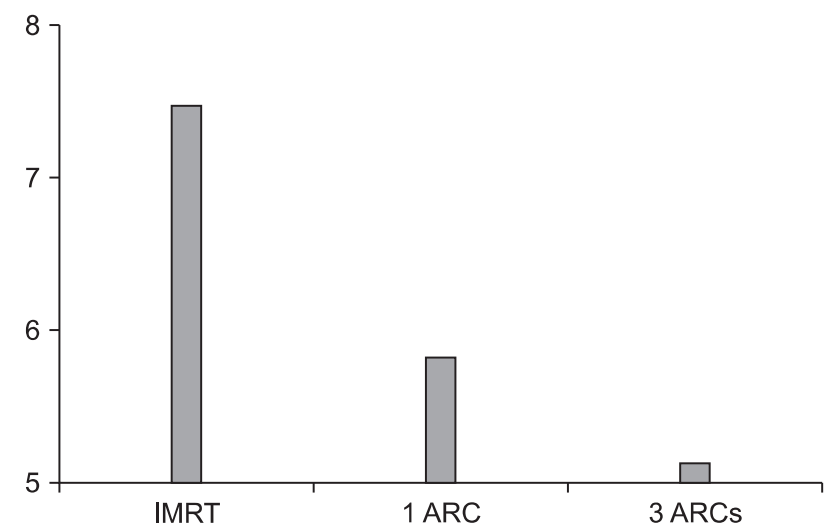

Fig. 6. Average gradient index. IMRT, intensity modulated radiation therapy.

plans in terms of the HI (Fig. 7).

For the aforementioned variables, the statistical significance (based on the $p$-value score) of the difference between the plans, is summarized in Table 1.

\section{Discussion and Conclusion}

Prostate cancer is a site in which highly conformal therapies have gained widespread use secondary to the organs at risk in the area and the benefit seen with dose escalation in metaanalysis [1]. In many large centers, IMRT-based plans have become standard for patients with prostate cancer. Although treatment volumes for those patients affected with low and intermediate risk prostate cancer have reached a general consensus, treatment of high-risk prostate cancer continues to be a controversial topic. The debate regarding the radiation therapy field is still ongoing and the inclusion of the pelvic

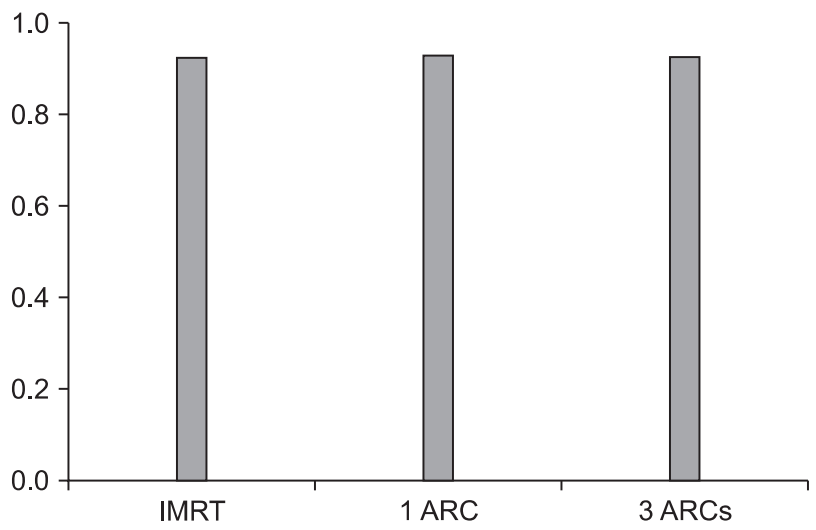

Fig. 7. Average homogeneity Index. IMRT, intensity modulated radiation therapy.

lymph nodes remains. Radiation toxicity in those patients receiving high-doses to both the prostate and pelvic lymph nodes has been noted with even lower doses than currently used [2]. We studied sixteen patients with high-risk prostate cancer as defined by NCCN to evaluate the target volume adequacy of coverage and efficiency as well as dose to OARs.

IMRT has been shown to improve coverage of the CTV by the prescription dose and reduced the volumes of the rectal and bladder walls significantly when compared to 3D conformal radiation therapy techniques in prostate cancer [3]. These benefits led to the widespread use of modulated therapy in prostate cancer and lead to the current standard of dose escalation [6]. VMAT represents a specific type of IMRT that was conceived to exploit increasing number of fields and decreasing treatment time $[7,8]$. By constantly changing MLC position, gantry position, and dose rate, VMAT has been shown to deliver equivalent plans in shorter time when compared with IMRT [9].

VMAT has been proposed to result in reduced treatment times and monitor units when compared to IMRT [10]. It has been shown to decrease the number of MUs required [11] There has been a hypothesized benefit in reducing secondary malignancies due to the reduction of interleaf scatter [12]. Additionally, arc therapy with multiple arcs allows for flexibility of dosage, increased sparing of normal tissue, and increased conformality $[13,14]$. VMAT, specifically multiple arcs, has been shown in head and neck cancer to provide better PTV dose homogeneity and similar or better OAR sparing $[15,16]$. VMAT, specifically multiple arcs, has also been shown to provide comparable PTV coverage in spine stereotactic body radiation therapy with greater efficiency compared to IMRT [17] 
Enhanced sparing of the rectum has been achieved with VMAT in prostate cancer patients undergoing dose escalation to an intraprostatic lesion [18].

We investigated the feasibility of using multiple static fields IMRT to single arc and 3 arcs IMAT in patients with highrisk prostate cancer. All three plans were comparable as far as adequacy of coverage. We chose the RTOG $\mathrm{Cl}$ to report on the target volume coverage. The conformity indices were 1.17, 1.20, and 1.15 for IMRT, $1 A R C$, and $3 A R C$, respectively; with 3ARC exhibiting slightly higher conformity. The conformity indices reported by Yoo et al. [19] and associates were 1.19, 1.25, and 1.20 for IMRT, single arc, and 2 arcs, respectively. Arc therapy was significantly better than IMRT but no significant difference among the one versus 2 arcs. The Gl, or "dose fall off" measure, also revealed an improvement with arc therapy in comparison to IMRT plans. $\mathrm{V}_{50}$-the volume covered by the $50 \%$ isodose line was significantly higher with the IMRT plan in comparison to the IMAT. $D_{\text {MEAN }}$ for organs at risk was lower with IMRT in comparison to IMAT plans and was comparable between $1 A R C$ and $3 A R C$. There was no significant difference between the three plans as far as the $D_{\text {MEAN }}$ of bladder. Our results are suggesting that VMAT offer more conformal plans in high-risk prostate cancer patients with minimal change in dose to organs at risk.

Efficiency of treatment was also improved in our cohort of patients when comparing IMRT versus arc therapy, with reductions in "Beam ON" time and number of MU. The average BOT decreased 4.6 and 2.8 minutes with the single arc and multiple arcs IMAT, respectively, plans compared to IMRT. The benefits of reduced "Beam ON" time include faster treatment time for the patient, which may result in greater patient comfort and increased number of patients to be treated on a machine. Also, with treatment given in a shorter amount of time, probability of intrafractional movement decreases. Average MU decreased significantly with the IMAT plans compared to IMRT; lowest number was achieved with 1ARC.

VMAT appears to improve treatment efficiency, dosimetry, and conformity for patients with high-risk prostate cancer when compared to IMRT. This could translate into increased patient quality of life and linear accelerator productivity.

\section{Conflict of Interest}

No potential conflict of interest relevant to this article was reported.

\section{References}

1. Viani GA, Stefano EJ, Afonso SL. Higher-than-conventional radiation doses in localized prostate cancer treatment: a meta-analysis of randomized, controlled trials. Int J Radiat Oncol Biol Phys 2009;74:1405-18.

2. Lawton CA, DeSilvio M, Roach M 3rd, et al. An update of the phase III trial comparing whole pelvic to prostate only radiotherapy and neoadjuvant to adjuvant total androgen suppression: updated analysis of RTOG 94-13, with emphasis on unexpected hormone/radiation interactions. Int J Radiat Oncol Biol Phys 2007;69:646-55.

3. Zelefsky MJ, Fuks Z, Happersett L, et al. Clinical experience with intensity modulated radiation therapy (IMRT) in prostate cancer. Radiother Oncol 2000;55:241-9.

4. Marks LB, Yorke ED, Jackson A, et al. Use of normal tissue complication probability models in the clinic. Int J Radiat Oncol Biol Phys 2010;76(3 Suppl):S10-9.

5. Radiation Therapy Oncology Group. RTOG Clinical Trials [Internet]. Philadelphia, PA: RTOG; c2013 [cited 2013 May 23] Available from: http://www.rtog.org/ClinicalTrials/Protocol Table.aspx.

6. Zelefsky MJ, Fuks Z, Hunt M, et al. High-dose intensity modulated radiation therapy for prostate cancer: early toxicity and biochemical outcome in 772 patients. Int J Radiat Oncol Biol Phys 2002;53:1111-6.

7. Pirzkall $A$, Carol MP, Pickett $B$, Xia P, Roach M 3rd, Verhey $\sqcup$. The effect of beam energy and number of fields on photonbased IMRT for deep-seated targets. Int J Radiat Oncol Biol Phys 2002;53:434-42.

8. Otto K. Volumetric modulated arc therapy: IMRT in a single gantry arc. Med Phys 2008;35:310-7.

9. Oliver M, Ansbacher W, Beckham WA. Comparing planning time, delivery time and plan quality for IMRT, RapidArc and Tomotherapy. J Appl Clin Med Phys 2009;10:3068.

10. Wolff D, Stieler F, Welzel G, et al. Volumetric modulated arc therapy (VMAT) vs. serial tomotherapy, step-and-shoot IMRT and 3D-conformal RT for treatment of prostate cancer. Radiother Oncol 2009;93:226-33.

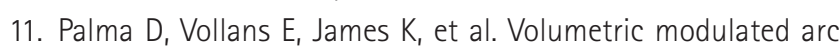
therapy for delivery of prostate radiotherapy: comparison with intensity-modulated radiotherapy and three-dimensional conformal radiotherapy. Int J Radiat Oncol Biol Phys 2008;72: 996-1001.

12. Hall EJ, Wuu CS. Radiation-induced second cancers: the impact of 3D-CRT and IMRT. Int J Radiat Oncol Biol Phys 2003;56:83-8.

13. Kopp RW, Duff M, Catalfamo F, Shah D, Rajecki M, Ahmad K. VMAT vs. 7-field-IMRT: assessing the dosimetric parameters of prostate cancer treatment with a 292-patient sample. Med 
Dosim 2011;36:365-72.

14. Tang G, Earl MA, Luan S, Wang C, Mohiuddin MM, Yu CX. Comparing radiation treatments using intensity-modulated beams, multiple arcs, and single arcs. Int J Radiat Oncol Biol Phys 2010;76:1554-62.

15. Verbakel WF, Cuijpers JP, Hoffmans D, Bieker M, Slotman BJ, Senan S. Volumetric intensity-modulated arc therapy vs. conventional IMRT in head-and-neck cancer: a comparative planning and dosimetric study. Int J Radiat Oncol Biol Phys 2009;74:252-9.

16. Alvarez-Moret J, Pohl F, Koelbl O, Dobler B. Evaluation of volumetric modulated arc therapy (VMAT) with Oncentra MasterPlan for the treatment of head and neck cancer. Radiat Oncol 2010;5:110.
17. Wu OU, Yoo S, Kirkpatrick JP, Thongphiew D, Yin FF. Volumetric arc intensity-modulated therapy for spine body radiotherapy: comparison with static intensity-modulated treatment. Int J Radiat Oncol Biol Phys 2009;75:1596-604.

18. Ost $P$, Speleers $B$, De Meerleer $G$, et al. Volumetric arc therapy and intensity-modulated radiotherapy for primary prostate radiotherapy with simultaneous integrated boost to intraprostatic lesion with 6 and $18 \mathrm{MV}$ : a planning comparison study. Int J Radiat Oncol Biol Phys 2011;79:920-6.

19. Yoo S, Wu OU, Lee WR, Yin FF. Radiotherapy treatment plans with RapidArc for prostate cancer involving seminal vesicles and Iymph nodes. Int J Radiat Oncol Biol Phys 2010;76:93542. 